\title{
A SaaS-based Software Modeling for Bank Intermediary Business
}

\author{
Bo Li ${ }^{1,2, *}$, Wei-Tek Tsai ${ }^{2}$, Haiying Zhou ${ }^{1}$, Decheng Zuo ${ }^{1}$ \\ ${ }^{1}$ Computer Science and Technology Department, Harbin Institute of Technology, China \\ ${ }^{2}$ School of Computing, I\&D Systems Engineering, Arizona State University Tempe, USA
}

Copyright (C) 2015 Horizon Research Publishing All rights reserved.

\begin{abstract}
Software-as-a-Service (SaaS) is a new research orientation for developing software, and has multi-tenancy architecture and customization features, which are very suitable for performance and benchmark test of OLTP transactions. And Bank Intermediary Business (BIB) is the most important business of Bank financial system. This paper focuses on establishing the SaaS-based BIB performance and benchmark architecture and proposes the SaaS-based BIB Database Model (SaaS-BIB-DM), the architecture layer (SaaS-BIB-AL), the data flow view (SaaS-BIB-DF) and the representative transaction model (SaaS-BIB-TM). The database is further extended with the SaaS hybrid two-layer partition methodology and the performance is proved to be better than that in three-tier $\mathrm{C} / \mathrm{S}$ architecture. And the specific SaaS-based BIB architecture which we proposed is 4-level SaaS-based architecture. Based on the analysis the state-of-art of BIB and SaaS, the paper further investigates future trend of SaaS-based performance testing architecture and benchmark.
\end{abstract}

Keywords SaaS, Performance, Benchmark, Testing, Modeling, HPFT, OLTP, SOA, MTA, BIB

\section{Introduction}

The rapid development in society and economy propose more performance requirements for HPFT (HighPerformance Fault-Tolerant) computers in national key industries, which makes the evaluation of HPFT performance and requirements for typical OLTP (On-Line Transaction Processing) applications becoming an important issue for both producers and end users. As SaaS (Softwareas-a-service) is arising to be a new research orientation and interesting aspects, the combination research of SaaS and OLTP is necessary. SaaS has the multi-tenancy architecture, specific partition schema that is horizon, vertical or mixed/hybrid database partition schema, and customization. With these features, research on database design/partition, scalability, recovery and continuous testing is becoming more and more important. SaaS is a new approach for developing software, and it is characterized by its multi-tenancy architecture and its ability to provide flexible customization to individual tenant. The most important features of SaaS are MTA (Multi-Tenancy Architecture) and customization, which create many new issues in software and testing fields. SaaS is software that deployed over the Internet and often run on a cloud platform. With SaaS, a software provider licenses an application to customer as a service on demand, through a subscription or a "pay-as-you-go" model. [6]

BIB is short for Bank Intermediary Business. BIB is developed as typical On-Line Transaction Processing (OLTP) transactions and provides various services to customers and third partner companies, such as On-Contract business, Trade-Charge agency, Trade-Payment agency, Multi-Trade business etc. BIB system has three actors, which is bank (service provider), third-party Company (multiple service consumers and users) and user (individual service consumer). Research indicates that the business model, database model, transaction/frame model, testing architecture and performance metrics constitute the benchmark and we analyze the BIB in following sections. BIB is one of the most important bank business assets and liability business, such as salary payment agency, water/electric fee charge agency, mobile fee agency etc. BIB has a large amount of data workload and average processing transactions, small transaction response time and high safety requirements. [9] BIB has three participants, which are third party client, client and bank. Bank-driven transactions are the main and key ones for BIB. We analyze the workflow of BIB and propose a new Bank Intermediary Four-Level Architecture. Layer1 is the GUI (Graphic User Interface) layer, different tenant (client or third-party client) has different GUI, and user can define its own style GUI panel. Layer2 is the workflow business process layer, which composed of three- tier of old BIBmodel (Bank Intermediary Business Model) transaction model, third-party client and client will process indirectly by the bank agency software. Layer3 is the services and composition layer, which composed of various BIB transactions, including 11 BIB transactions, such as 
New-Contract, Modify-Contract, Cancel-Contract, TracePayment, Trade-Charge, Balance-Lookup, Contract-Lookup, Trade-Lookup, Data-Maintain, Trade-Cleanup and TradeRecord Transactions. We will describe these architectures and transactions specific in Section 3. Layer4 is the database level, we use SaaS hybrid database partition schema here to enhance the performance of database and testing.

This paper is organized as follows. Firstly, the paper introduces the state-of-art of SaaS, BIB and performance testing schemas. Section 2 summarizes the related works. Section 3 proposes a new Bank Intermediary Four-Level Architecture. Section 4 gives the scenario and use case study. Section 5 comes to a conclusion, to provide a scalable framework of BIB based on SaaS.

\section{Study of SaaS and BIB}

SaaS is software that deployed over the Internet and/or is deployed to run behind a firewall on a local-area network or personal computer. [2] From software providers' view, SaaS is the software, which licenses the application to customer as a service on demand, through a subscription or a "pay-as-you-go" model and meanwhile has multi-tenant and customization features. Multi-tenant means that the SaaS can be used by multiple users simultaneously but do not interrupt or influence each other. MTA (Multi-tenancy architecture) refers to a principle where a single instance of the software runs on a server, serving multiple client organization or tenants. A realistic SaaS application needs to address multiple issues, such as scalability, database partitioning and consistency, fault-tolerant aspects, security and fairness, parallel processing, isolation, performance and availability simultaneously. From end user's view, SaaS is feeling like using the software individually which is not shared with other tenants.

Recent studies about benchmarks and measurements techniques have been developed to quality and evaluate HPFT system performance, such as TPC-C [10] and TPC-E [11] benchmarks, which are the most significant OLTP benchmark and proposed by TPC council in 90th. TPC-C [10] benchmark simulates an order-entry computing environment where a population of users executes transactions against the TPC-C simple database and TPC-E [11] benchmark models a brokerage firm with customers who generate transactions related to trades, account inquiries, and market research etc. Although there are many OLTP benchmarks, there is still a lack of benchmarks that is based on actual transactions and using high-performance architectures. The development tendency of BIB (Bank Intermediary Business) in around the world, especially in the difficult economy and financial environment, gives rise to study in the BIB benchmark fields. BIB has large amount of data workload and average processing transactions, small transaction response time and high safety requirements. [9] The most important performance metrics to measure OLTP system is the performance of system, which is discussed in our previous paper [9][12], such as response time, system utilization, transactions per second. Performance benchmarks must include two major components, a workload which represents the work that the system must perform during the benchmark run time and a set of performance measures that characterizes the performance of the system under benchmark testing. The basis idea is to model the BIB business's architecture, database and transactions in order to establish the real BIB models and propose the suitable BIB benchmark with qualified metrics.

\section{A SaaS-based BIB Architecture and Models}

We categorize HPFT computer performance testing methods into three classes, methods based on performance benchmarks such as OLTP TPC serials, methods based on workload testing tools such as HP LoadRunner and Spirent Avalanche and methods based on architecture and workload/workflow analysis, which is studied in this paper. BIBmodel (Bank Intermediary Business Model) is modeled by the key-processing modules of BIB business. In this section, we propose and design the SaaS-based architecture, SaaS-BIB-DM (Database Model), SaaS-BIB-DF (DataFlow) and transactions description.

\section{A. A SaaS-based BIB Architecture}

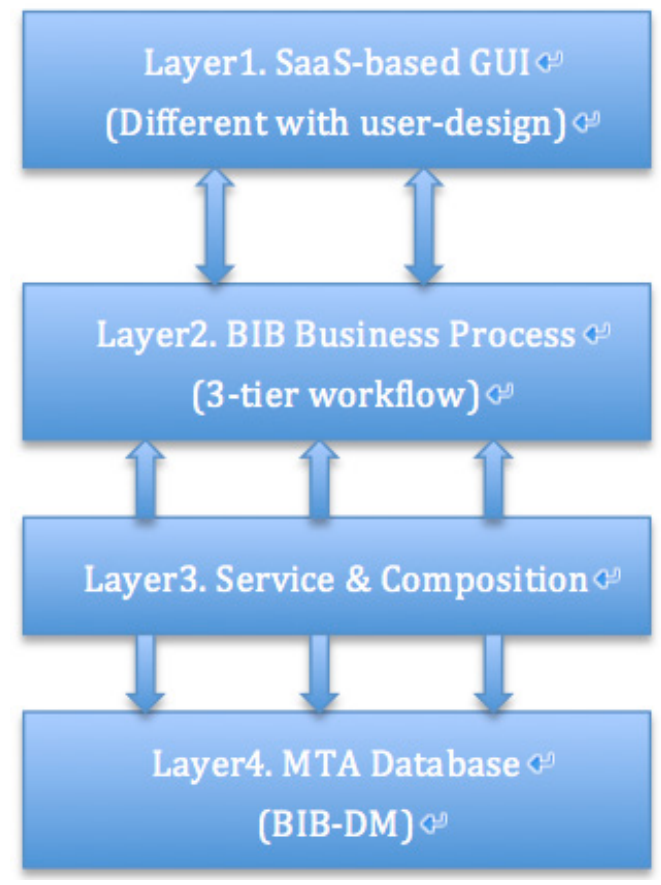

Figure 1. A SaaS-based BIB Architecture.

Testing comprises activities that validate a system's aspects. New challenges arise at each SaaS testing level and typical SOA (Service-Oriented Architecture) testing is composed of composition testing, integration testing and functional testing. But the most fundamental is the 
architecture and models. We divide this SaaS-based BIB architecture into four layers, which are layer1 GUI (different users has different GUI (Graphic User Interface) according to the user-design requirements), layer2 BIB business process (description of BIB workflow, such as agent-in workflow, agent-out workflow, lookup-workflow etc.), layer3 Services and Compositions (each BIB services is constructed separately and provides services for the upper layer) and layer4 database (propose an BIB-DM which is database model of Bank Intermediary Business with the hybrid SaaS partition principles ). The specific SaaS-based $\mathrm{BIB}$ architecture is showed in figure 1.

\section{B. SaaS-BIB-DM (Database Model)}

Table 1. Database Tables Description

\begin{tabular}{|c|c|c|c|c|}
\hline Category & Description & Table Name & Prefix & Num \\
\hline \multirow[t]{3}{*}{$\begin{array}{l}\text { Partner } \\
\text { Tables }\end{array}$} & $\begin{array}{l}\text { Basic Info of } \\
\text { Partner }\end{array}$ & Partner_Info & $\mathrm{PI}_{-}$ & 11 \\
\hline & Account of Partner & Partner Account & $\mathrm{PA}$ & 5 \\
\hline & Salary of Employee & Employee_Salary & ES & 3 \\
\hline \multirow{2}{*}{$\begin{array}{l}\text { Customer } \\
\text { Tables }\end{array}$} & Info of Customers & Customer Info & $\mathrm{Cl}$ & 10 \\
\hline & $\begin{array}{l}\text { Account } \\
\text { Customer }\end{array}$ & Customer_Account & $\mathrm{CA}_{-}$ & 9 \\
\hline \multirow{13}{*}{$\begin{array}{l}\text { Bank } \\
\text { Tables }\end{array}$} & $\begin{array}{l}\text { Basic Info of } \\
\text { Channel }\end{array}$ & Channel_Info & $\mathrm{CH}_{-}$ & 3 \\
\hline & Types of Channel & Channer Type & $\mathrm{CT}$ & 3 \\
\hline & $\begin{array}{l}\text { Counter Channel } \\
\text { Info }\end{array}$ & Counter_Channel & $\mathrm{CH}_{-}$ & 3 \\
\hline & ATM Channel Info & ATM Channel & $\mathrm{CH}$ & 3 \\
\hline & $\begin{array}{l}\text { Call } \\
\text { Channel }\end{array}$ & Call_Channel & $\mathrm{CH}_{-}$ & 3 \\
\hline & $\begin{array}{ll}\begin{array}{l}\text { Online } \\
\text { Channel }\end{array} & \text { Bank } \\
\end{array}$ & Online_Chennel & $\mathrm{CH}_{-}$ & 3 \\
\hline & Product Info Details & Product_Info & PR & 8 \\
\hline & $\begin{array}{l}\text { Business } \\
\text { Transaction }\end{array}$ & Business_Info & BI_ & 8 \\
\hline & Contract Info & Contract & $\mathrm{CN}$ & 19 \\
\hline & $\begin{array}{l}\text { Teller Permission } \\
\text { Info }\end{array}$ & Teller_Permission & TP_ & 5 \\
\hline & Bank Agency Info & Agency Info & $\mathrm{Al}$ & 6 \\
\hline & Business Serial Info & Business Serial & BS & 6 \\
\hline & $\begin{array}{l}\text { Process Control } \\
\text { Info }\end{array}$ & Process_Control & $\mathrm{PC}_{-}$ & 6 \\
\hline \multirow{3}{*}{$\begin{array}{l}\text { Dimension } \\
\text { Tables }\end{array}$} & Zip Code Info & Zip Code & $\mathrm{ZC}$ & 2 \\
\hline & Product Type Info & Product Type & PT & 2 \\
\hline & Types of Business & Business Type & BT & 2 \\
\hline
\end{tabular}

MTA is short for Multi-Tenant Architecture and it is the key feature of SOA (Service-Oriented Architecture). MTA has the following benefits: (1). Accelerate time to value; (2).always on the latest release;(3).you can control your technology adoption;(4).turn the OR into AND;(5).and community collaboration is high; The SaaS-BIB-DM is abbreviation of Software-as-a- Service based Bank Intermediary Business Database Model, which described in the layer4 of the SaaS-based BIB Architecture. We use the SaaS hybrid database partition schema to enhance the performance of database and testing. We divide the database of BIB into four categories, including Partner Tables (descriptions of third-party company tables), Customer Tables (descriptions of personal account and information tables), Bank Tables (descriptions of bank channel, account, contract and other information tables) and Dimension Tables (descriptions of assistance or accessories). There are three types of data in the SaaS-BIB- DM model, which is metadata, actual data and pivot index. And the database tables' description and the definition of database tables are showed in table 1.

Many partitioning schemes have been studied and we propose a hybrid partition scheme for the SaaS-BIB-DM model, which divide the table into rows and horizontal partitioning the key-value stores.

\section{SaaS-BIB-WF (Workflow)}

The SaaS-BIB-WF is abbreviation of Software-as-aService based Bank Intermediary Business Workflow, which described in the layer2 of the SaaS-based BIB Architecture. The workflow business is composed of three- tier of old BIBmodel's (Bank Intermediary Business Model) actors, including bank, third-party company client and personal client. The workflow is showed in figure 2 and figure 3.

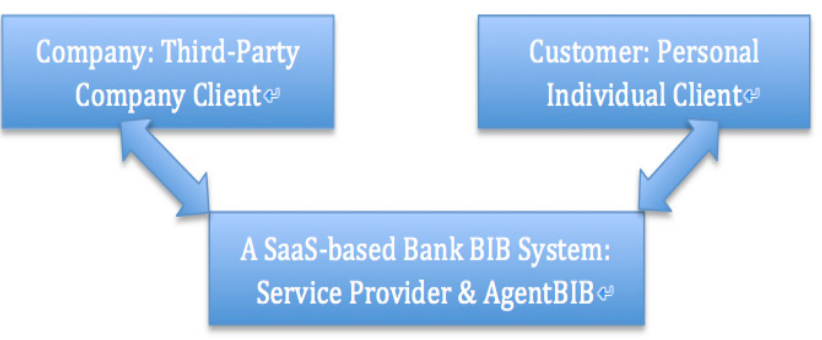

Figure 2. A 3-tier service provider \& service consumer \& service broker model.

The SaaS-BIB-WF (Workflow) is composed of seven steps and if transform step failed, the workflow will start over from step1. The workflow starts from the evocation of third-party Company or personal client required the BIB (Bank Intermediary Business) through bank counter or Internet services. Once the bank receives the requirements (processing the agent-transactions), the bank will search the corresponding services and transform to the SaaS-BIB-WF on-demand form. If the Success judgment is failed, it goes back to step1. Then step4 will process them utilizing the layer3 of SaaS-BIB architecture. Next, the real lookup and process workflow execution and simultaneously record the testing results and generate the results \& reports for further analysis.

\section{SaaS-BIB-TM (Transaction Model)}

The SaaS-BIB-TM is abbreviation of Software-as-aService based Bank Intermediary Business Transaction Model, which described in the layer3 of the SaaS-based BIB Architecture. The services and composition layer is composed of various BIB transactions, including 12 BIB transactions, such as New-Contract, Modify-Contract, Cancel-Contract, Trade-Payment, Trade-Charge, BalanceLookup, Contract-Lookup, Trade-Lookup, DataMaintenance, Trade-Cleanup, Trade-Record and SaaS-Trace Transactions. 


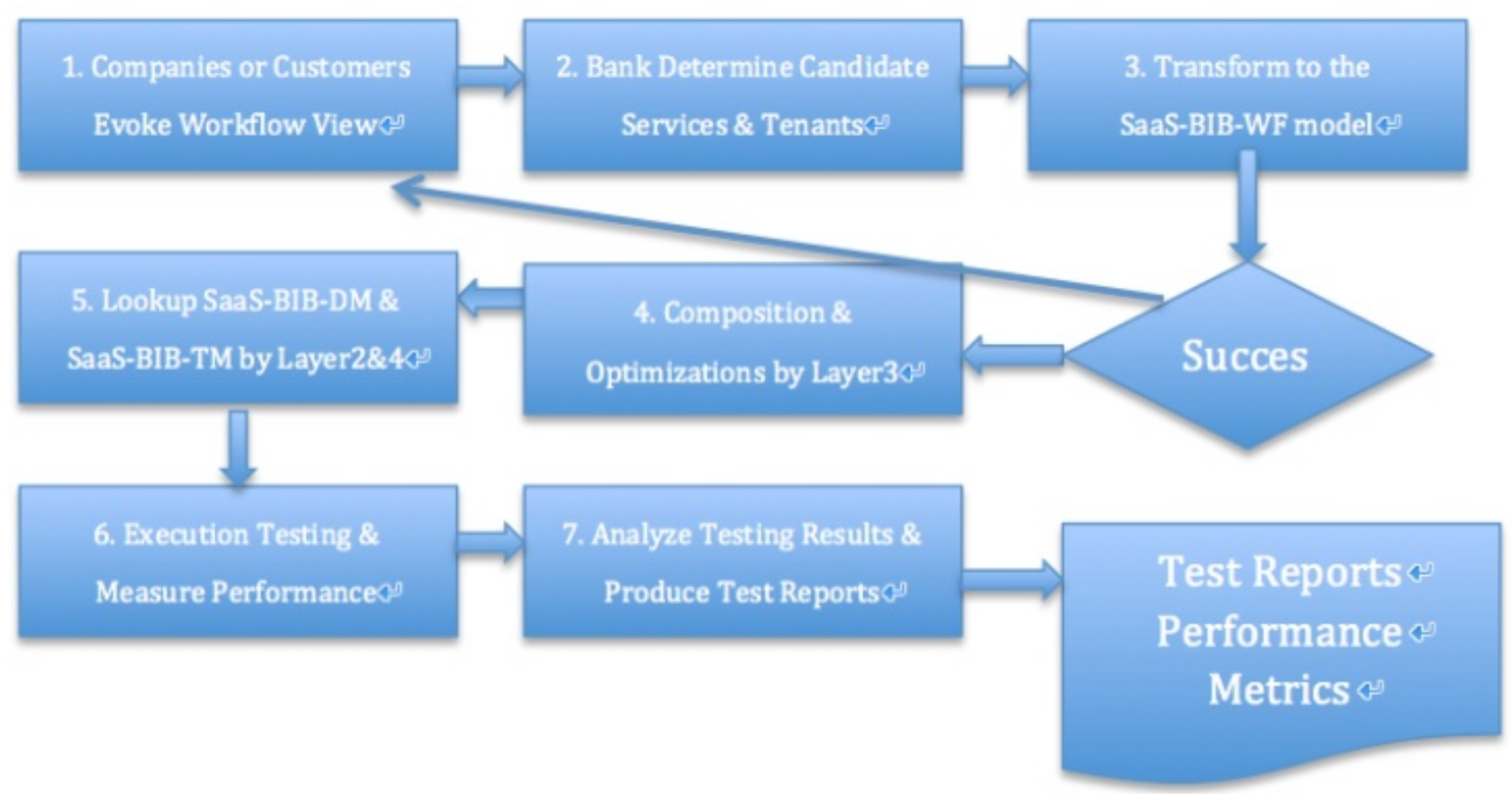

Figure 3. The SaaS-BIB-WF Workflow Figure.

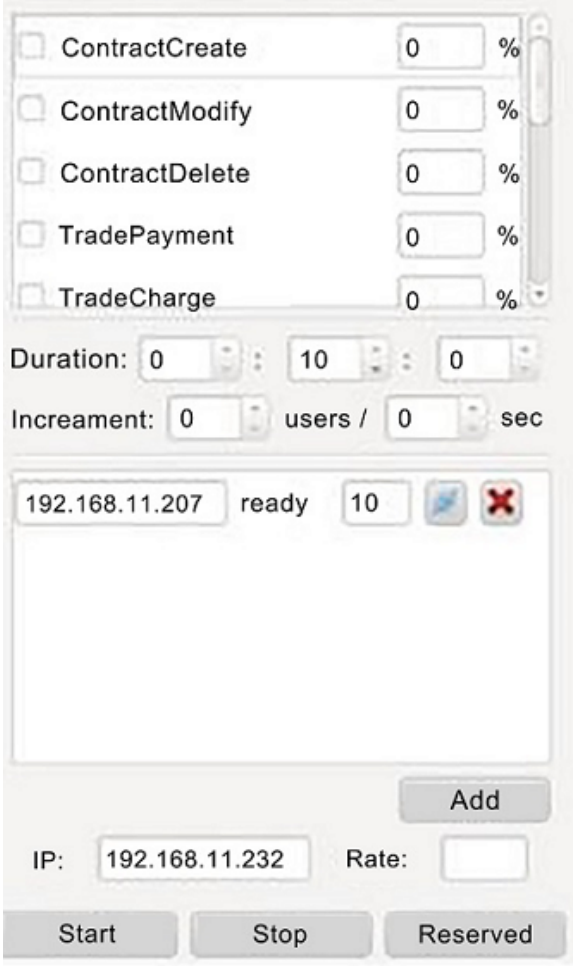
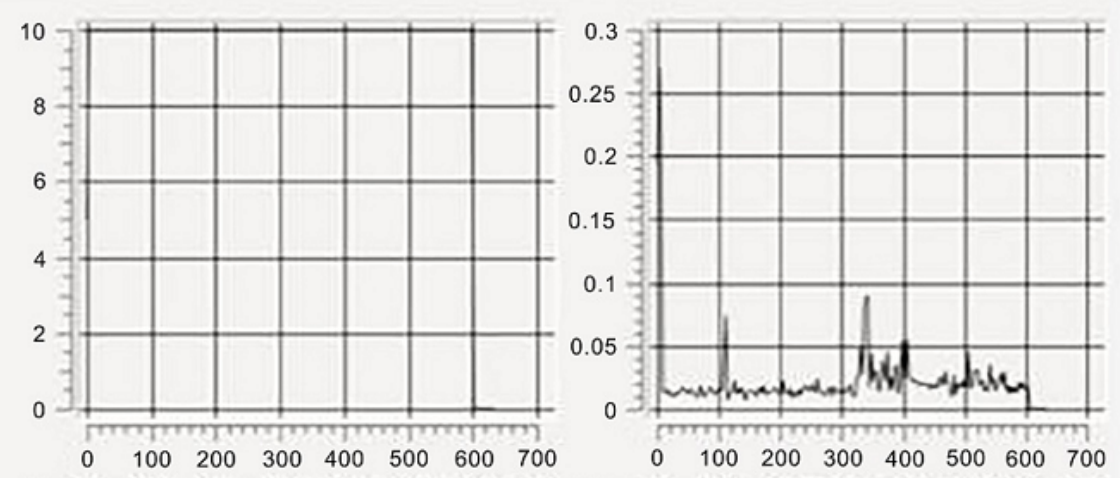

Figure 4. Example of a Saas-based Performance Testing Runtime GUI

\section{BIB Transaction Description and Test Case Study}

Before In this section, we first introduce two representative scenarios for Bank Intermediary Business (BIB) to illustrate the nucleus transactions, and each scenario has three participants. And then we explain our designed transactions for configuration and development acquirements.

\section{A. BIB Transaction Description}

We consider the most common BIB business, which is Trade-Charge and Trade-Payment. These trade transaction means that the bank is the agency to process financial or 
account problems for third-party company clients and personal clients.

There are four categories transactions, which have $12 \mathrm{BIB}$ transitions. Contract transactions including three veritable transactions are New-Contract, Modify-Contract and Cancel-Contract. The premise of each transaction is that the third-party company client or personal client has already signed a required contract with the bank tier. These Contract category transactions are the fundamental of every Lookup, or Trade or Trace transactions. The Trade category has two key processing transactions, Trade-Charge and TradePayment transaction. The former transaction is applicable for scenario 1 and the latter transaction is applicable for scenario 2 for execution and testing. The Lookup category has three various lookup processing based on different target. Precisely, Balance-Lookup is to inquire the account balance of given customer, Contract-Lookup is to inquire whether the customers or clients have signed these kind of contracts with certificate bank tier, and Trade-Lookup is to inquire the $\log$ or trace of requested trade. The Trace category is to record some useful information or documents for execution these transactions or frames. Above all, the SaaS-based BIB benchmark at least has these four metrics: tpsBank (transactions per second of bank business), PCN (Parallel Concurrent Number of users), TRT (Transaction Processing Response Time) and RU(Resource utilization, including memory-RU, disk-RU, CPU-RU and bandwidth- RU), which are described in our previous paper and showed in figure 4.

\section{B. Test Case Study}

A scenario is a sequence of activities connected by the four operators: sequence, choice, loop and concurrency. Each activity is a data assignment, exchanging an event, doing an action, or executing a sub-scenario. This paper considers about two different scenarios in the future test case study:

(1).Simple scenario 1, the scenario of Trade-Charge Transaction, which is a telephone company, has multiple users who want to pay the mobile phone bills through the bank intermediary business. Then this telephone company will sign a contract with the bank to authorize the bank intermediary business system charging the fees of its mobile users. Instead of go to telephone company to pay the bills each time, users of the telephone company can choose using enormous bank agency to pay the bills which is very convenient since the bank has many bank counter and Internet services are mature and security enough.

(2).Complex or composite scenario 2 is that an international company has to pay salaries each month to its employee and send out the bonus yearly. The international company should sign a contract with the bank intermediary business system to get the service of agency pay salary workflow. According to the contract, it will have a pay salary date and the BIB system will update the balance of the company and the accounts of its employees' simultaneously. This transaction should be done together or done nothing and it is the scenario of Trade-Payment Transaction.

\section{Conclusion and Future Works}

SaaS is characterized by its multi-tenancy architecture and its ability to provide flexible customization to individual tenant, and SaaS is developed in much realistic OLTP business, especially in BIB (Bank Intermediary Business) applied with this new SaaS architecture. We firstly combine the SaaS architecture with a typical OLTP business - BIB. And we propose a SaaS-based BIB architecture, database model, transaction model and workflow model. For case study, we describe two representative scenarios for execution and deployment. These models are the fundamental and essential elements of a representative benchmark.

In conclusion, the complexity of transactions is increasing due to new business offerings, compliance and consolidation, which makes the $4 \mathrm{C}$ testing and schema, becomes very important, including Complete \& Collaborative Testing, Continuous Validation and Constraint Virtualization. So, for the future study, we will continuous study the $4 \mathrm{C}$ of the architecture and BIB business and obtain some useful testing results to fulfill the schema and theory.

\section{Acknowledgements}

The authors would like to thank all the colleagues and co-partners who have contributed to the study. The work is supported by the grants from the National High-Tech R \& D Plan of China (2009AA01A404), International S \& $T$ Cooperation Program of China (No. 2010DFA14400). Also thanks to the visiting scholarship of CSC and thanks to the support of Harbin Institute of Technology, Arizona State University and Tsinghua University.

\section{REFERENCES}

[1] Benchmark Characteristics and Benchmark Performance Prediction[C]." 1996 ACM 0734-2071/96/1100-0344. Vol. 14 No. 4. Pp. 334-384

[2] Wei-Tek Tsai, Qihong Shao, Yu Huang, Xiaoying Bai. "Towards a Scalable and Robust Multi-tenancy SaaS[C]." 2010.

[3] Xiaoying Bai, Muyang Li, Bin Chen, Wei-Tek Tsai, Jerry Gao. "Cloud Testing Tools[C]." SOSE2011.

[4] Brian Davis. "iTKO Overview[C]". Interactive TKO, Inc. Jan. 2009.

[5] K. Kanoun, P. Koopman. "Dependability Benchmarking: A Realit or A Dream[C].” IEEE Computer Society. 2008:1.

[6] Wei-Tek Tsai, Yu Huang, Qihong Shao, and Xiaoying Bai. "Data Partitioning and Redundancy Management for Robust 
Multi-Tenancy SaaS[J].” Int J Software Informatics, Vol. 4, No. 4, December 2010. pp. 437-471.

[7] Wei-Tek Tsai, Qihong Shao, Wu Li. "OIC: Ontology- based Intelligent Customization Framwork for SaaS[C].” 2010.

[8] Wu Li, Yann-Hang Lee, Wei-Tek Tsai. "Service- Oriented smart home applications: composition, code generation, deployment and execution[C]." SOCA 2011.

[9] Bo Li, Haiying Zhou, Decheng Zuo, Zhan Zhang, Peng Zhou and Long Jia. "Performance Modeling and Benchmarking of Bank Intermediary Business on High- Performance Fault-Tolerant Computers[C]." DSN2011. 978-1-4577-0375-1/11 pp. 234-239.

[10] Transaction Processing Performance Council (TPC). TPC-C
BENCHMARKTM Standard Specification Version 5.11 .2010

[11] Transaction Processing Performance Council (TPC). TPC-E BENCHMARKTM Standard Specification Version 1.23.0.2010J. Clerk Maxwell, A Treatise on Electricity and Magnetism, 3rd ed., vol. 2. Oxford: Clarendon, 1892, pp.6873.

[12] Bo Li, Haiying Zhou, Decheng Zuo. "Workload Performance Characterizaiton and Test Strategy of High- Performance Fault-Tolerant Computers based on BIBbench[J]." ICMEE2011.

[13] Wei-Tek Tsai, Xiaoying Bai \& Yu Huang. "Software-as-a-Service (SaaS): Perspectives and Challenges". Science China. May 2012 Vol. 53 No. 1:1-18 